\title{
Overexpression of RGPR-p117 induces the decrease in protein and DNA contents in cloned normal rat kidney proximal tubular epithelial NRK52E cells
}

\author{
SUSUMU TOMONO, NATSUMI SAWADA and MASAYOSHI YAMAGUCHI \\ Laboratory of Endocrinology and Molecular Metabolism, Graduate School of Nutritional Sciences, \\ University of Shizuoka, 52-1 Yada, Suruga-ku, Shizuoka 422-8526, Japan
}

Received January 23, 2007; Accepted February 27, 2007

\begin{abstract}
A novel protein RGPR-p117 was discovered as a regucalcin gene promoter region-related protein that binds to the TTGGC motif. Regucalcin is known to regulate the intracellular signaling system in many cell types. RGPR-p117 has been shown to enhance the promoter activity of the regucalcin gene in cloned normal rat kidney proximal tubular epithelial NRK52E cells. The role of RGPR-p117 in cell function remains to be elucidated, however. This study was undertaken to determine whether overexpression of RGPR-p117 has an effect on cell proliferation, protein and DNA contents in NRK52E cells. NRK52E cells (wild-type) or stable RGPRp117/phCMV2-transfected cells (transfectants) were cultured in Dulbecco's minimum essential medium containing 5\% bovine serum (BS). RGPR-p117 was markedly expressed in the transfectants. NRK52E cells (wild-type) or transfectants were cultured for 24,48 , or $72 \mathrm{~h}$ in a medium containing $5 \%$ BS, and after subconfluency the cells were cultured for 24 , 48 , or $72 \mathrm{~h}$ in a medium without BS. Cell proliferation was not significantly changed in the transfectants as compared with that of wild-type cells. Protein and DNA contents in NRK52E cells were significantly decreased in the transfectants with cell proliferation in the presence of BS. When NRK52E cells with subconfluency were cultured for 24,48 , or $72 \mathrm{~h}$ in a medium without BS, the number of transfectant cells was not significantly changed compared with that of wild-type cells. Protein and DNA contents in NRK52E cells were significantly decreased in the transfectants cultured in a medium without BS after subconfluency. This study demonstrates that overexpression of RGPR-p117 induces the decrease
\end{abstract}

Correspondence to: Dr M. Yamaguchi, Laboratory of Endocrinology and Molecular Metabolism, Graduate School of Nutritional Sciences, University of Shizuoka, 52-1 Yada, Suruga-ku, Shizuoka 422-8526, Japan

E-mail: yamaguch@u-shizuoka-ken.ac.jp

Key words: RGPR-p117, regucalcin, DNA, protein, NRK52E cells in protein and DNA contents in NK52E cells, indicating its role in the regulation of cell function.

\section{Introduction}

RGPR-p117 was discovered as a regucalcin gene promoter region-related protein $(1,2)$. Regucalcin has been demonstrated to play a multifunctional role as a regulatory protein in the intracellular signaling system of many cell types (reviewed in refs. 3-6). RGPR-p117 is conserved in various vertebrate species, including the human, rat, mouse, bovine, rabbit, and chicken $(1,2)$, indicating a great conservation of RGPR-p117 genes throughout evolution. The entire human RGPR-p117 cDNA consists of $3,989 \mathrm{bp}$, which contains an open reading frame (ORF) of 3,180 bp, encoding a protein of 1,060 amino acid residues $(1,2)$. A comparison of human RGPR-p117 cDNA sequence with the genomic sequence database indicates that the gene consists of at least 26 exons spanning $\sim 42 \mathrm{~kb}$ and is localized in human chromosome, 1q25.2 (1).

RGPR-p117 mRNA is ubiquitously expressed in many rat tissues (7), and is stably expressed for physiological changes in the rat liver (8). Mammalian RGPR-p117 conserves a leucine zipper motif $(1,2)$. Moreover, the computer analysis of subcellular localization of RGPR-p117 from six vertebrates shows the probability of nuclear localization at $>52.2 \%$; the nuclear localization in the rat and mouse is $78.3 \%$ (1).

RGPR-p117 is suggested to have a role in nuclear function. RGPR-p117 has been shown to localize in the nuclei of cloned normal rat kidney proximal tubular epithelial NRK52E cells (9). Overexpression of RGPR-p117 can enhance the regucalcin promoter activity that is related to the NF-1 consensus sequences including the TTGGC motif, and its enhancing effect is partly mediated through phosphorylation and dephosphorylation of proteins in NRK52E cells (10-12). RGPR-p117 has been demonstrated to enhance the expression of the regucalcin gene in the nucleus of kidney cells, indicating its role as a transcription factor. The role of RGPR-p117 in the regulation of cellular function, however, remains to be elucidated.

This study was undertaken to determine whether overexpression of RGPR-p117 has an effect on cellular function in cloned normal kidney tubular epithelial NRK52E cells We found that overexpression of RGPR-p117 induces the decrease in protein and DNA contents in NRK52E cells. 


\section{Materials and methods}

Chemicals. Non-essential amino acid solution, bovine serum (BS) and penicillin-streptomycin solution $(5,000 \mathrm{U} / \mathrm{ml}$ penicillin; $5,000 \mu \mathrm{g} / \mathrm{ml}$ streptomycin) were obtained from Gibco Laboratories (Grand Island, NY). Dulbecco's modified Eagle's medium (DMEM) was obtained from Sigma Chemical (St. Louis, MO). Other chemicals were purchased from Wako Pure Chemical Co. (Osaka, Japan). Reagents used were dissolved in distilled water or ethanol, and some reagents were passed through ion-exchange resin to remove metal ions.

Anti-RGPR-p117 IgG. Rabbits (Japan SLC Inc., 2.5-3.0 kg), used to raise anti-rat RGPR-p117 antibodies, were subcutaneously injected with $5.0 \mathrm{mg}$ per animal of antigen emulsified with Freunds' complete adjuvant two times, at 2 -week intervals. The antigen contained the amino acid sequences of CQMLGRPKSFIPSFQV (605-620) and GKNTKSSGFGWFSW (901-914) in rat RGPR-p117 sequences (1). These sequences had a high homology for human RGPRp117. Animals were sacrificed with bleeding 24 days after the last injection to obtain antiserum. The antiserum was purified by Protein A column (Nygen Co., Yonkers, NY, USA) to obtain anti-RGPR-p117 IgG.

RGPR-p117 transfectants. The expression plasmid for HA-tagfused RGPR-p117, HA-RGPR-p117/phCMV2, was constructed as described previously (9). First, HA-RGPR-p117/phCMV2 was digested with $X c m I$ and NotI to obtain the XcmI-NotI (229-3174) fragment. Second, the upstream and the Nterminal regions of RGPR-p117 were amplified by PCR using two primers: ACTGCAGCATAACAACCTCAAGACCCAAAG (PstI site and -46 to -25) and GAGTATGGGTGACTGGGATAAGATCC (243-265), and rat liver cDNA library as a template. The amplified DNA fragment was cloned into the pGEMT vector, and the DNA sequence was confirmed by DNA sequencing. This plasmid was digested by PstI and $X c m \mathrm{I}$, and then two DNA fragments (Pst I-XcmI and XcmINotI) were cloned into the Pst I site and NotI site of the phCMV2 expression vector (27). The resultant plasmid was designated as RGPR-p117/phCMV2 (28).

For transient transfection assay, the NRK52E cells were grown on $35-\mathrm{mm}$ dishes to $\sim 70 \%$ confluence. Each RGPRp117/phCMV2 and phCMV2 vector alone was transfected into NRK52E cells using the synthetic cationic lipid component, Lipofectamine, according to the manufacturer's instructions (Invitrogen) (9). Forty-eight hours after transfection, cells were harvested and used for subsequent experiments. NRK52E cells were transfected with RGPRp117/phCMV2 vector, alone using Lipofectamine. After $24 \mathrm{~h}$, neomycin ( $1.0 \mathrm{mg} / \mathrm{ml}$ geneticin G418, Sigma) was added to the cultures for selection, and cells were plated at a limiting dilution. Multiple surviving clones were isolated, transferred to $35-\mathrm{mm}$ dishes, and grown in the medium without neomycin. RGPR-p117 was stably expressed in the transfectants. In the experiments, the transfectants were cultured for 24-72 $\mathrm{h}$ in DMEM containing 5\% BS.

Cell culture. The cloned normal rat kidney proximal tubular epithelial NRK52E cells $\left(1 \times 10^{5}\right.$ cells $)(13,14)$ were maintained for $72 \mathrm{~h}$ in DMEM supplemented with 5\% BS, non-essential amino acid, and $50 \mathrm{U} / \mathrm{ml}$ penicillin and $50 \mu \mathrm{g} / \mathrm{ml}$ streptomycin in humidified $5 \% \mathrm{CO}_{2} / 95 \%$ air at $37^{\circ} \mathrm{C}$ to obtain subconfluent monolayers. After culturing, cells were washed three times with phosphate-buffered saline (PBS), and the cells were incubated for 24,48 , or $72 \mathrm{~h}$ in DMEM without $5 \%$ BS.

Cell counting. After trypsinization of each of the culture dishes using $0.2 \%$ trypsin plus $0.02 \%$ EDTA in $\mathrm{Ca}^{2+} / \mathrm{Mg}^{2+}$ free phosphate-buffered saline (PBS) for $2 \mathrm{~min}$ at $37^{\circ} \mathrm{C}$, cells were collected and centrifuged in PBS at $100 \mathrm{x}$ g for $5 \mathrm{~min}$. The cells were re-suspended in PBS solution and stained with eosin. Cell numbers were counted under a microscope using a hemocytometer plate. For each dish, we took the average of two countings.

Western blot analysis. NRK52E cells and the transfectants cultured with $10 \%$ FBS were washed three times with PBS after culturing. The pooled cells were scraped in ice-cold $0.25 \mathrm{M}$ sucrose $(0.5 \mathrm{ml})$ and disrupted for $30 \mathrm{sec}$ with an ultrasonic device. Scraped cells were also homogenized in a Potter-Elvehjem homogenizer with a Teflon pestle. The homogenates were spun at 5,500 $\mathrm{x} g$ in a refrigerated centrifuge for $5 \mathrm{~min}$. The $5,500 \mathrm{x}$ g supernatant was pooled to analyze RGPR-p117 protein using Western blot analysis (15). Protein concentration in the $5,500 \mathrm{x} g$ supernatant of the cell homogenate was determined by the method of Lowry et al (16).

Aliquots of protein $(20 \mu \mathrm{g})$ were mixed with $5 \mathrm{X}$ Laemmli sample buffer, boiled for $5 \mathrm{~min}$, and SDS-PAGE was performed by the method of Laemmli (15) using $12 \%$ polyacrylamide resolving gel. After SDS-PAGE, the proteins were then transferred onto a polyvinylidene difluoride membrane $(100 \mathrm{~mA})$ for $3 \mathrm{~h}$. The membranes were incubated with a polyclonal rabbit anti-RGPR-p117 antibody, which was diluted 1:2,000 in $10 \mathrm{mM}$ Tris- $\mathrm{HCl}, \mathrm{pH} 8.0$, containing $150 \mathrm{mM} \mathrm{NaCl}, 0.1 \%$ (w/v) Tween-20 (washing buffer), and $5 \%(\mathrm{w} / \mathrm{v})$ skim milk for $1 \mathrm{~h}$. The membranes incubated with antibody were washed four times with washing buffer. Then the membranes were incubated for $1 \mathrm{~h}$ with horseradish peroxidase-linked anti-rabbit IgG which was diluted 1:5,000 with washing buffer containing $5 \%(\mathrm{w} / \mathrm{v})$ skim milk, and washed again. Detection of the protein bands was performed for 20 min using an enhanced chemiluminescent kit following the manufacturer's instructions. The molecular size of the detecting protein was determined by running standard protein molecules of known sizes in parallel.

Analytical procedures. To determine the protein concentration in NRK52E cells, the cells were washed 3 times with PBS, scraped into $0.5 \mathrm{ml}$ of ice-cold $0.25 \mathrm{M}$ sucrose solution, and disrupted for $30 \mathrm{sec}$ with an ultrasonic device. Protein concentration in the cell homogenate was determined by the method of Lowry et al (16) and expressed as the amount of protein $(\mu \mathrm{g})$ per dish.

To measure DNA content in the cells, the cells were detached by using $0.2 \%$ trypsin plus $0.02 \%$ EDTA in $\mathrm{Ca}^{2+} / \mathrm{Mg}^{2+}$-free PBS and washed with PBS. The cells were shaken with $2.0 \mathrm{ml}$ of ice-cold $0.1 \mathrm{~N} \mathrm{NaOH}$ solution for $6 \mathrm{~h}$ after disruption. After alkali extraction, the samples were 


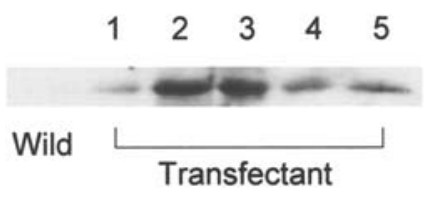

Figure 1. Expression of RGPR-p117 in the cloned normal rat kidney proximal tubular epithelial NRK52E cells (wild-type) and stable RGPRp117/phCMV2 transfectants. The RGPR-p117 content of multiple neomycinresistant clones was analyzed by immunoblotting with an anti-RGPR-p117 antibody. Lanes 1-5, RGPR-p117/phCMV2-transfected clones. Western blot analysis was carried out on the extracts ( $20 \mu \mathrm{g}$ of the 5,500-g supernatant of the cell homogenate) obtained from the NRK52E cells per lane. The figure shown is representative of four experiments.

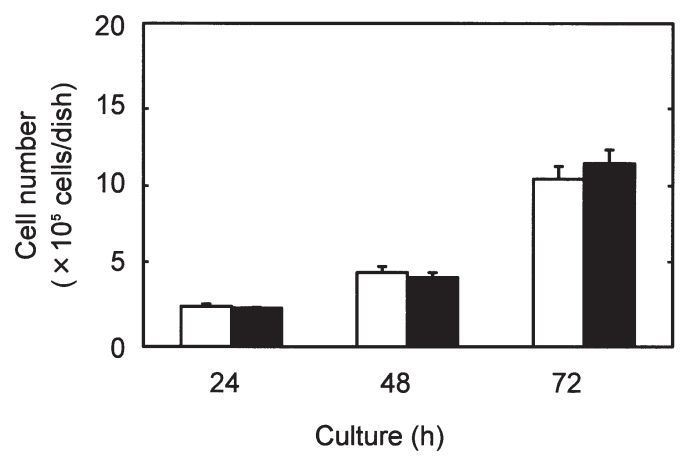

Figure 2. Cell proliferation of the cloned normal rat kidney proximal tubular epithelial NRK52E cells (wild-type) and stable RGPR-p117/phCMV2 transfectants. Cells $\left(1 \times 10^{5}\right)$ were cultured for 24,48 , or $72 \mathrm{~h}$ in the presence of $5 \%$ BS. Each value is the mean \pm SEM of four experiments. White bars, wild-type cells; black bars, transfectants.

centrifuged at $10,000 \mathrm{xg}$ for $5 \mathrm{~min}$, and the supernatant was determined by the method of Ceriotti (17) and expressed as the amount of DNA $(\mu \mathrm{g})$ per dish.

Statistical analysis. Data were expressed as the mean \pm SEM. Statistical differences were analyzed using the Student's t-test. $\mathrm{P}$-values $<0.05$ were considered to indicate statistically significant differences. The ANOVA multiple comparison test was used to compare the treatment groups.

\section{Results}

Generation of the NRK52E cell line overexpressing RGPRp117. To generate transfectants with stably overexpressed RGPR-p117 in NRK52E cells, phCMV2 vector- or RGPRp117/phCMV2-transfected NRK52E cells were cultured in neomycin-containing medium. Multiple neomycin-resistant clones were selected, and the RGPR-p117 content in these clones was analyzed by immunoblotting with anti-RGPR-p117 antibody. The generation of transfectants with anti-RGPRp117 was determined using Western blot analysis (Fig. 1). The RGPR-p117 content in RGPR-p117/phCMV2-transfected clones (lanes 1-5) was markedly increased compared with that of the parental wild-type NRK52E cells. Among these clones, clone 2 expressed the highest RGPR-p117. This cell line (transfectant) was used for subsequent experiments.

Change in protein and DNA contents in RGPR-p117overexpressing NRK52E cells with proliferation. The cloned

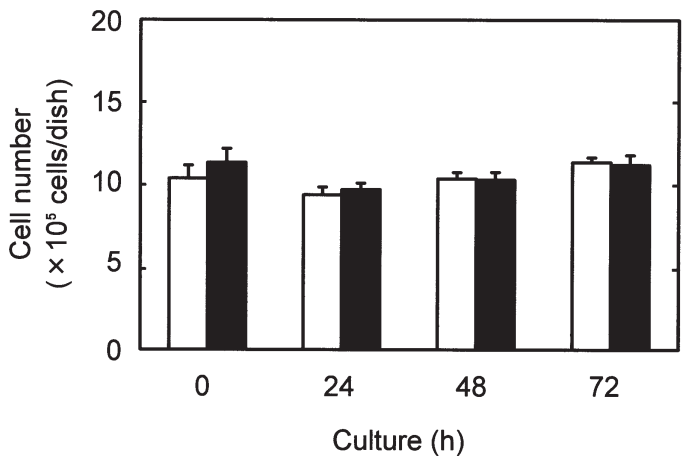

Figure 3. Change in protein in the cloned normal rat kidney proximal tubular epithelial NRK52E cells (wild-type) and stable RGPR-p117/phCMV2 transfectants. Cells $\left(1 \times 10^{5}\right)$ were cultured for 24,48 , or $72 \mathrm{~h}$ in the presence of $5 \%$ BS. Each value is the mean \pm SEM of six experiments. " $p<0.05$ or ${ }^{* *} \mathrm{p}<0.01$ compared with the control value of wild-type cells. White bars, wild-type cells; black bars, transfectants.

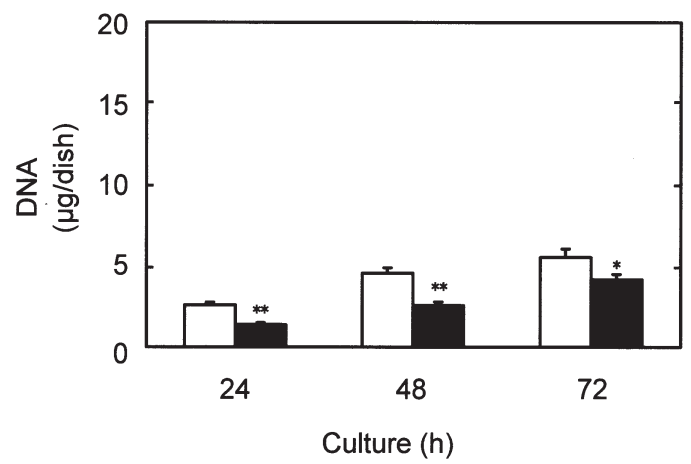

Figure 4. Change in DNA content in the cloned normal rat kidney proximal tubular epithelial NRK52E cells (wild-type) and stable RGPR-p117/ phCMV2 transfectants. Cells $\left(1 \times 10^{5}\right)$ were cultured for 24,48 , or $72 \mathrm{~h}$ in the presence of $5 \%$ BS. Each value is the mean \pm SEM of six experiments. ${ }^{*} \mathrm{p}<0.05$ or ${ }^{* *} \mathrm{p}<0.01$ compared with the control value of wild-type cells. White bars, wild-type cells; black bars, transfectants.

normal rat kidney proximal tubular epithelial NRK52E cells (wild-type) and stably RGPR-p117/phCMV2-transfected NRK52E cells were cultured in a medium containing $5 \%$ BS for 24,48 , or $72 \mathrm{~h}$ to reach subconfluent monolayers. Cell proliferation of the transfectants was not significantly changed compared with that of NK52E wild-type cells (Fig. 2), and also was unaltered in NRK52E cells (mocktype) transfected with the phCMV2 vector (data not shown).

The protein (Fig. 3) or DNA (Fig. 4) content in NK52E cells (wild-type) was significantly decreased in the transfectants overexpressing RGPR-p117, when the wild-type cells or transfectants were cultured for 24,48 , or $72 \mathrm{~h}$ in a medium containing $5 \% \mathrm{BS}$.

Change in protein and DNA contents in RGPR-p117overexpressing NRK52E cells after subconfluency. The cloned normal rat kidney proximal tubular epithelial NRK52E cells (wild-type) and stably RGPR-p117/phCMV2transfected NRK52E cells were cultured in a medium containing 5\% BS for $72 \mathrm{~h}$. After subconfluency, the cells were cultured for 24,48 , or $72 \mathrm{~h}$ in a medium without BS. The number of NRK52E cells was not significantly 


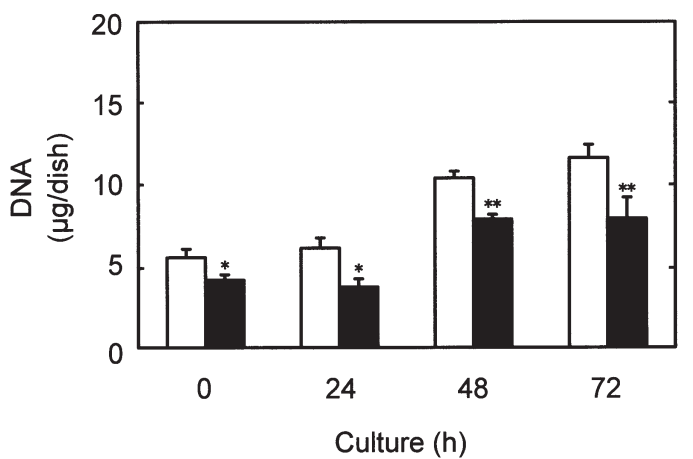

Figure 5. Cell number of the cloned normal rat kidney proximal tubular epithelial NRK52E cells (wild-type) and stable RGPR-p117/phCMV2 transfectants. Cells $\left(1 \times 10^{5}\right)$ were cultured for 24,48 , or $72 \mathrm{~h}$ in the presence of $5 \%$ BS. Each value is the mean \pm SEM of six experiments. White bars, wild-type cells; black bars, transfectants.

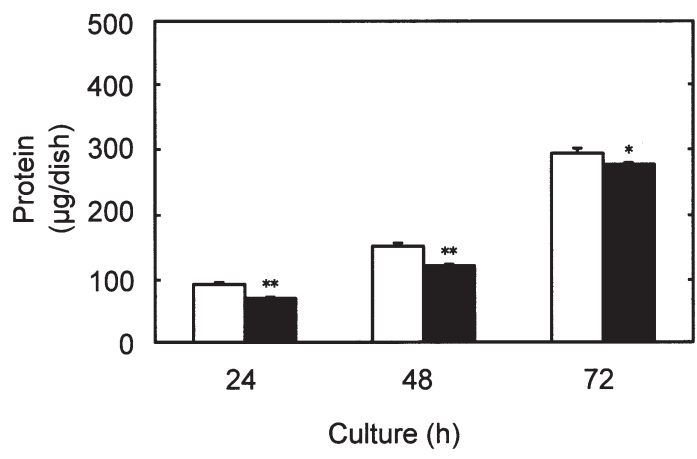

Figure 6. Change in protein in the cloned normal rat kidney proximal tubular epithelial NRK52E cells (wild-type) and stable RGPR-p117/phCMV2 transfectants. Cells $\left(1 \times 10^{5}\right)$ were cultured for $72 \mathrm{~h}$ in the presence of $5 \% \mathrm{BS}$, and the cells with subconfluency were cultured for 24,48 , or $72 \mathrm{~h}$ in medium without BS. Each value is the mean \pm SEM of six experiments. ${ }^{*} \mathrm{p}<0.05$ or ${ }^{* *} \mathrm{p}<0.01$ compared with the control value of wild-type cells. White bars, wild-type cells; black bars, transfectants

changed in the transfectants as compared with the wild-type cells (Fig. 5).

Protein (Fig. 6) or DNA (Fig. 7) content in NRK52E cells was significantly decreased in the transfectants overexpressing RGPR-p117. These decreases were greater with extended periods of culturing.

\section{Discussion}

The role of RGPR-p117 in the regulation of cellular function has not been clarified. RGPR-p117 is suggested to play a role in nuclear function in cloned rat kidney proximal tubular epithelial NRK52E cells. RGPR-p117 has been found to localize in the cytoplasm and nucleus with immunocytochemical and Western blot analysis using HA-RGPRp 117/phCMV2-transfected NRK52E cells (9). Overexpression of RGPR-p117 enhances the mRNA expression and protein levels of regucalcin, which plays a multifunctional role as a regulatory protein in intracellular signaling systems in many cell types (3-6), and in NRK52E cells (10-12). RGPR-p117 may play a role as a transcriptional factor related to the TTGGC motif $(11,12)$.

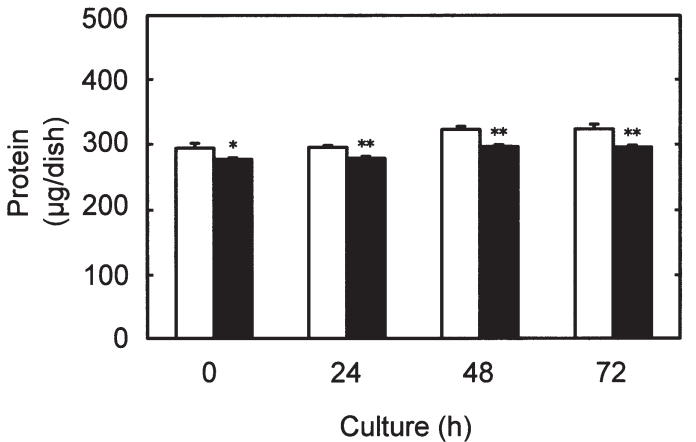

Figure 7. Change in DNA in the cloned normal rat kidney proximal tubular epithelial NRK52E cells (wild-type) and stable RGPR-p117/phCMV2 transfectants cultured without BS after subconfluency. Cells $\left(1 \times 10^{5}\right)$ were cultured for $72 \mathrm{~h}$ in the presence of $5 \% \mathrm{BS}$, and the cells with subconfluency were cultured for 24,48 , or $72 \mathrm{~h}$ in medium without BS. Each value is the mean \pm SEM of six experiments. ${ }^{*} \mathrm{p}<0.05$ or ${ }^{* *} \mathrm{p}<0.01$ compared with the control value of wild-type cells. White bars, wild-type cells; black bars, transfectants.

We found that overexpression of RGPR-p117 caused a significant decrease in protein and DNA contents in the cloned normal rat kidney proximal tubular epithelial NRK52E cells. This was a novel finding for the role of RGPR-p117 in cells.

Overexpression of RGPR-p117 did not cause a significant change in the proliferation of NRK52E cells cultured in the presence of BS including many hormones and cytokines, indicating that RGPR-p117 does not have an effect on cell proliferation. When the NRK52E cells with subconfluency were cultured in a medium without BS, the number of transfectant cells was not significantly changed with overexpression of regucalcin. This result indicates that RGPR-p117 is not involved in the induction of apoptosis.

Overexpression of RGPR-p11171 induced a significant decrease in protein and DNA contents in NRK52E cells, although the number of cells was not significantly changed. It is speculated that overexpression of RGPR-p117 has a suppressive effect on protein and DNA synthesis in NRK52E cells. It is possible that overexpression of RGPR-p117 may cause the degradation of protein and DNA in NRK52E cells. Alternatively, RGPR-p117 may have a role in the regulation of protein and DNA contents in NRK52E cells.

RGPR-p117 has been shown to localize in the cytoplasm and nucleus with immunocytochemical analysis using HARGPR-p117/phCMV2-transfected NRK52E cells (9). The results with Western blotting for HA-RGPR-p117 showed that RGPR-p117 was localized in the plasma membranes, nucleus, mitochondria, microsomes, and cytoplasm of the transfectants (9). These observations suggest that RGPR-p117 has many functions in NRK52E cells. RGPR-p117 may have a regulatory effect on DNA synthesis in the nucleus and on protein synthesis in the translational process in the cytoplasm and microsomes of NRK52E cells. Furthermore, this remains to be elucidated.

In conclusion, it has been demonstrated that overexpression of RGPR-p117 does not have an effect on cell proliferation in cloned normal rat kidney proximal tubular epithelial NRK52E cells, and that it induces a decrease in protein and DNA contents in NRK52E cells. 


\section{References}

1. Misawa $\mathrm{H}$ and Yamaguchi $\mathrm{M}$ : Molecular cloning and sequencing of cDNA coding for a novel regucalcin promoter region-related protein in rat, mouse, and human liver. Int $\mathrm{J}$ Mol Med 8: 513-520, 2001.

2. Sawada $\mathrm{N}$ and Yamaguchi $\mathrm{M}$ : A novel regucalcin promoter region-related protein: Comparison of nucleotide and amino acid sequences in vertebrate species. Int J Mol Med 15: 97-104, 2005.

3. Yamaguchi $\mathrm{M}$ : A novel $\mathrm{Ca}^{2+}$-binding protein regucalcin and calcium inhibition. Kohama K (ed). Japan Sci Soc Press, Tokyo and CRC Press. Boca Raton, pp19-41, 1992.

4. Yamaguchi M: Role of regucalcin in calcium signaling. Life Sci 66: 1769-1780, 2000.

5. Yamaguchi M: The role of regucalcin in nuclear regulation of regenerating liver. Biochem Biophys Res Commun 276: 1-6, 2000.

6. Yamaguchi $\mathrm{M}$ : Role of regucalcin in maintaining cell homeostasis and function (Review). Int J Mol Med 15: 371-389, 2005.

7. Misawa $\mathrm{H}$ and Yamaguchi M: Gene expression for a novel protein RGPR-p117 in various species: The stimulation by intracellular signaling factors. J Cell Biochem 87: 188-193, 2002.

8. Yamaguchi M, Misawa $\mathrm{H}$ and Ma ZJ: Novel protein RGPRp117: The gene expression in physiologic state and the binding activity to regucalcin gene promoter region in rat liver. J Cell Biochem 88: 1092-1100, 2003.

9. Sawada N, Nakagawa T, Murata T and Yamaguchi M: Nuclear localization of novel protein RGPR-p117 in normal rat kidney proximal tubular epithelial cells. Int J Mol Med 16: 809-814, 2005.
10. Sawada N and Yamaguchi M: Overexpression of RGPR-p117 enhances regucalcin gene expression in cloned normal rat kidney proximal tubular epithelial cells. Int J Mol Med 16: 1049-1055, 2005.

11. Sawada N and Yamaguchi M: Overexpression of RGPR-p117 enhances the regucalcin gene promoter activity in cloned normal rat kidney proximal tubular epithelial cells: Involvement of TTGGC motif. J Cell Biochem 99: 589-597, 2006.

12. Sawada $\mathrm{N}$ and Yamaguchi M: Involvement of nuclear factor I-AI in the regulation of regucalcin gene promoter activity in cloned normal rat kidney proximal tubular epithelial cells. Int J Mol Med 18: 665-671, 2006.

13. Rice EK, Tesck GH, Cao Z, Cooper ME, Metz CN, Bucala R, Atkins RC and Nikillc-Peterson DJ: Induction of MIF synthesis and secretion by tubular epithelial cells: a novel action of angiotensin II. Kidney Int 63: 1265-1275, 2003.

14. Nakagawa $\mathrm{T}$ and Yamaguchi $\mathrm{M}$ : Hormonal regulation on regucalcin mRNA expression in cloned normal rat kidney proximal tubular NRK52E cells. J Cell Biochem 95: 589-597, 2005 .

15. Laemmli UK: Cleavage of structural proteins during the assembly of the head of bacteriophage T4. Nature 224: 680-685, 1970.

16. Lowry OH, Rosebrough NH, Farr AL and Randall RF: Protein measurement with the Folin phenol reagent. J Biol Chem 193: 265-273, 1951.

17. Ceriotti G: Determination of nucleic acids in animal tissues. J Biol Chem 241: 59-70, 1955. 